\title{
Prevalência de fatores de risco de doença cardiovascular em trabalhadores de condominios
}

\author{
Prevalence of cardiovascular disease risk factors \\ among condominium workers
}

\author{
Maurício Susumu Osawa', Mariana Ragassi Urbano², Ana Beatryz Prenzier Suzuki
}

\begin{abstract}
RESUMO|Contexto: As doenças cardiovasculares (DCV) representam a principal causa de morte no Brasil e existem poucos estudos que relacionam seus fatores de risco com trabalhadores de condomínios. Objetivos: Determinar a prevalência dos principais fatores de risco para as doenças cardiovasculares em trabalhadores de condomínios residenciais e comerciais e relacioná-los com seus enquadramentos funcionais. Métodos: Estudo epidemiológico transversal e observacional com 380 indivíduos selecionados aleatoriamente por enquadramento funcional: portaria, zeladoria, faxina e auxiliar de limpeza. Foi utilizado um formulário individual padronizado para coleta de dados após consulta médica em ambulatório de Medicina Geral, que atende 4.735 trabalhadores em Londrina (PR). Os fatores de risco para DCV analisados foram: idade, sexo, índice de massa corpórea, hipertensão arterial sistêmica (HAS), diabetes mellitus tipo 2 (DM2), pré-diabetes (pré-DM), hipercolesterolemia, evento cardiovascular prévio, sedentarismo e tabagismo. Resultados: A maioria dos trabalhadores era composta por homens, exercia a função na portaria e apresentava mais de 40 anos de idade. As prevalências encontradas foram: HAS - 39,5\%; hiperglicemia (pré-diabetes e diabetes tipo 2) - 18,2\%; hipercolesterolemia - 35,3\%; tabagismo - 12,1\%. Cabe ressaltar a elevada prevalência de excesso de peso encontrada em mais $80 \%$ da amostra estudada, sendo que quase metade se situava na faixa de obesidade. Além disso, o sedentarismo foi encontrado em $80 \%$ dos indivíduos pesquisados. Conclusão: A distribuição elevada dos fatores de risco de DCV entre os trabalhadores de condomínio deve estimular a adoção de estratégias para melhorar as condições de saúde desses trabalhadores. O maior conhecimento do perfil clínico-ocupacional de um grupo fornece subsídios para viabilizar o correto direcionamento de recursos nos programas de saúde, bem como aumenta a qualidade de vida no trabalho.
\end{abstract}

Palavras-chave I doenças cardiovasculares; epidemiologia; fatores de risco; saúde do trabalhador.

ABSTRACT | Context: Cardiovascular diseases (CVD) are the leading cause of death in Brazil and there are few studies that relate their risk factors with condominium workers. Objectives: To determine the prevalence of the major risk factors for CVD in residential and commercial condominium workers and relate them to their functional frameworks. Methods: Transversal and observational epidemiologic study with 380 individuals randomly selected by functional framework: reception desk, concierge, housekeeping and cleaning assistant. A standardized form was used to collect data after medical consultation in a general practice clinic responsible for 4.735 workers in Londrina, state of Paraná, Brazil. The following risk factors for CVD were analyzed: age, sex, body mass index, systemic arterial hypertension (SAH), type 2 diabetes (T2DM), pre-diabetes (IGT - impaired glucose tolerance), hypercholesterolemia, previous cardiovascular event, sedentary lifestyle and smoking. Results: Most of the workers were men, worked as doormen and were more than 40 years old. Prevalence rates were: SAH - 39,5\%; hyperglycemia (type 2 diabetes and pre-diabetes) - 18,2\%; hypercholesterolemia - 35,3\%; smoking - 12,1\%. A high prevalence of being overweight was found in more than $80 \%$ of the sample, with almost half being in the obesity range. Moreover, $80 \%$ of the individuals were sedentary. Conclusion: The high distribution of CVD risk factors among condominium workers should encourage the adoption of strategies to improve their health. Greater knowledge of the clinical and occupational profile of a group provides subsidies to enable the correct targeting of resources in health programs and increases the quality of work life.

Keywords I cardiovascular diseases; epidemiology; risk factors; occupational health. 


\section{INTRODUÇÃO}

As doenças cardiovasculares (DCV) representam a maior causa de mortalidade no Brasil e no mundo. São responsáveis por cerca de $20 \%$ de todas as mortes em indivíduos acima de 30 anos $^{1}$. As DCV são ainda responsáveis por alta frequência de internações, ocasionando custos médicos e socioeconômicos elevados ${ }^{2}$. Dentre os fatores de risco, destacam-se: hipertensão arterial sistêmica (HAS), diabetes mellitus (DM), hipercolesterolemia, idade, sedentarismo e tabagismo ${ }^{3}$.

O controle de alguns dos fatores de risco pode reduzir a mortalidade secundária ocasionada por doenças ateroscleróticas. O controle da HAS diminui o acidente vascular cerebral (AVC) em cerca de $42 \%$ e a isquemia miocárdica em $15 \%{ }^{4}$. A redução do colesterol em cerca de $30 \%$ diminui o risco de infarto do miocárdio em $33 \%$, o AVC em $29 \%$ e a mortalidade cardiovascular em $28 \%{ }^{5}$. A cessação do tabagismo pode diminuir o risco de mortalidade em até $70 \% \mathrm{em}$ indivíduos já portadores de isquemia miocárdica ${ }^{6}$.

A HAS tem alta prevalência e baixas taxas de controle, é considerada um dos principais fatores de risco modificáveis e um dos mais importantes problemas de saúde pública ${ }^{7}$. Inquéritos populacionais em cidades brasileiras apontaram uma prevalência de HAS acima de $30 \%{ }^{8,9}$. Entre os gêneros, a prevalência foi de $35,8 \%$ nos homens e de $30 \%$ em mulheres, semelhante à de outros países ${ }^{10}$.

O DM é considerado uma epidemia em curso e fator de risco independente para $\mathrm{DCV}^{11}$. Mundialmente, 240 milhões de indivíduos apresentavam essa condição em 2005 e estimativas apontam que 366 milhões serão acometidos em 2030, com dois terços deles em países em desenvolvimento ${ }^{12}$. As crescentes incidência e prevalência não são atribuídas apenas ao envelhecimento populacional e aos avanços no tratamento da doença, mas, principalmente, ao estilo de vida atual, caracterizado por sedentarismo e hábitos alimentares que predispõem ao sobrepeso e obesidade.

A relação entre os níveis séricos elevados de colesterol com o desenvolvimento das DCV está embasada em diversas evidências epidemiológicas e clínicas, que demonstraram ser a hipercolesterolemia um fator de risco não só para o aparecimento da aterosclerose, mas também para a morte por causa coronariana ${ }^{13,14}$. Ensaios clínicos realizados em indivíduos hipercolesterolêmicos mostraram que a redução dos níveis de colesterol resulta numa significativa redução da mortalidade e de outros eventos cardiovasculares ${ }^{13,15}$.

A obesidade apresenta relação bem estabelecida com uma variedade de complicações para a saúde, como DM, dislipidemia e DCV. Quanto maior o excesso de peso, maior é a gravidade da doença ${ }^{16}$. Em 2010, foram divulgados dois grandes levantamentos dos números do excesso de peso e obesidade no Brasil: o VIGITEL Brasil 2009 e a Pesquisa de Orçamentos Familiares 2008-2009 (POF). Apesar de metodologias diferentes, ambas mostraram uma elevada frequência de excesso de peso: 42,3 e $48 \%$, respectivamente ${ }^{17,18}$. A POF revelou que a obesidade e o excesso de peso têm aumentado rapidamente nos últimos anos em todas as faixas etárias. Nesse levantamento, $50 \%$ dos homens e $48 \%$ das mulheres se encontram com excesso de peso, sendo que 12,5 e 16,9\% apresentam obesidade, respectivamente.

O sedentarismo foi identificado pela Organização Mundial da Saúde (OMS) como o quarto fator de risco mais importante para a mortalidade global (6\% das mortes), depois da HAS (13\%), do tabagismo (9\%) e do DM $(6 \%)^{19}$. Tem sido demonstrado que a atividade física regular reduz o risco de DCV, DM, HAS, câncer colorretal, câncer de mama e depressão. Ademais, a prática de exercícios físicos constitui peça-chave no gasto calórico e, portanto, é determinante no balanço energético e no controle de peso ${ }^{19-21}$.

O tabagismo representa a principal causa de morte evitável em todo o mundo. A OMS atribui 5 milhões de mortes por ano às doenças relacionadas ao tabagismo, muitas delas prematuramente ${ }^{22}$. Estimativas apontam que em 2030 o tabaco cause 8 milhões de mortes anuais, sendo metade delas em indivíduos em idade produtiva (entre 35 e 69 anos) e $80 \%$ em países em desenvolvimento ${ }^{23,24}$. O uso do tabaco é responsável por $90 \%$ dos casos de câncer pulmonar, $75 \%$ das bronquites crônicas e enfisemas e $25 \%$ das doenças isquêmicas do coração ${ }^{25}$. No Brasil, houve queda da proporção de fumantes na população. Em 1989, 34,8\% dos adultos eram tabagistas ${ }^{26}$, enquanto que, em 2008, a prevalência foi de $17,2 \%{ }^{27}$. Dados das capitais brasileiras e do Distrito Federal revelaram frequência de $16,2 \%$ de fumantes adultos no ano de 2006 e 14,8\% em $2011^{28}$.

Programas e ações em qualidade de vida no trabalho são medidas cada vez mais estudadas e implementadas no ambiente laboral, uma vez que sua inclusão gera redução de custos com a saúde dos colaboradores em decorrência da menor prevalência de doenças e aumento de produtividade ${ }^{29}$. 
A correta investigação e determinação dos problemas na população-alvo permite incorporar esses programas com maior eficácia e alocar adequadamente os investimentos em recursos físicos e humanos ${ }^{30}$.

As categorias de porteiros, zeladores e trabalhadores de limpeza apresentam grande relevância social, principalmente nas sociedades contemporâneas e mais urbanizadas.

Em decorrência da alta mortalidade por DCV e da escassez de estudos dos seus fatores de risco nessa população específica de trabalhadores, o presente estudo teve como objetivo avaliar esses fatores em trabalhadores de condomínios no município de Londrina (PR).

\section{MÉTODOS}

Trata-se de estudo transversal, descritivo, desenvolvido com dados clínicos de trabalhadores de condomínio atendidos em ambulatório de Medicina Geral localizado em Londrina (PR), Brasil. O ambulatório atende um total de 4.735 usuários ativos cadastrados.

No cálculo do tamanho da amostra utilizaram-se os seguintes parâmetros: prevalência de 50\% para os fatores de risco de doença cardiovascular na região, margem de erro de 5\% e confiabilidade e precisão da amostra em $95 \%$, o que resultou em 356 indivíduos.

Selecionaram-se dados de 380 indivíduos de ambos os sexos, que foram atendidos em ambulatório de Medicina Geral no período de julho a dezembro de 2014. As profissões de maior ocorrência foram estudadas: portaria, zeladoria, faxina e auxiliar de limpeza; as duas últimas agrupadas como serviços de limpeza.

Os dados foram coletados em formulário após realização da consulta pelo médico responsável do setor. Foram aferidos peso e altura e calculado o índice de massa corpórea (IMC). Os valores foram classificados em: peso normal (IMC >18,50 a $24,99 \mathrm{~kg} / \mathrm{m}^{2}$ ), sobrepeso (IMC $\geq 25$ a $29,99 \mathrm{~kg} / \mathrm{m}^{2}$ ) e obesidade (IMC $\geq 30 \mathrm{~kg} / \mathrm{m}^{2}$ ).

Hipertensão arterial sistêmica foi considerada naqueles indivíduos que já faziam uso de anti-hipertensivos ou conforme diagnóstico em atendimento médico.

Diabetes mellitus tipo 2 (DM2) foi considerado nos trabalhadores que já faziam tratamento para a doença ou naqueles que tiveram o diagnóstico confirmado durante avaliação médica por glicemia de jejum ou teste de tolerância oral à glicose. Para o diagnóstico de pré-diabetes (pré$\mathrm{DM})$, o indivíduo apresentava glicemia de jejum entre 100 e $125 \mathrm{mg} / \mathrm{dL}$ ou teste de tolerância oral à glicose com glicemia após 2 horas entre 140 e $200 \mathrm{mg} / \mathrm{dL}$.

A hipercolesterolemia foi identificada nos indivíduos que apresentavam níveis de colesterol total maior ou igual a $240 \mathrm{mg} / \mathrm{dL}$ ou se já eram usuários prévios de agente hipolipemiante (estatina ou fibrato).

Pesquisou-se no antecedente pessoal a ocorrência ou não de eventos cardiovasculares prévios: infarto agudo do miocárdio, AVC ou equivalente isquêmico, por exemplo, endarterectomia carotídea para tratamento de estenose de artéria carótida.

As variáveis relativas aos hábitos de vida foram tabagismo e atividade física. Para identificar se o trabalhador apresentava sedentarismo ou não, utilizou-se a definição da Organização Mundial da Saúde, que sugere que atividade física suficiente seja determinada como a prática de atividades aeróbias de moderada intensidade num período de 150 minutos por semana ou atividade física intensa em 75 minutos, ou a combinação de ambas ${ }^{31}$.

A análise estatística dos dados foi feita no software $R$ versão 3.2.0 (2015) $)^{32}$. Realizou-se uma análise exploratória para verificar as prevalências dos fatores de risco, seguida do teste $\chi^{2}$ ao nível de significância de 5\% para verificar a associação entre as ocupações estudadas e os fatores de risco de DCV.

\section{RESULTADOS}

A maioria dos trabalhadores que foram atendidos no ambulatório era composta por homens, exercia a função na portaria e tinha mais de 40 anos de idade (Tabela 1).

A prevalência geral da HAS foi de $39,5 \%$. Dentre as ocupações avaliadas, variou entre 31,91 e $51,85 \%$, valores esperados ao se considerar estudos em diferentes regiões brasileiras e em subgrupos ${ }^{33}$.

As situações clínicas de hiperglicemia, representadas por pré-DM e DM2, tiveram prevalência de 7,4 e 10,8\%, respectivamente. As taxas encontradas seguem o panorama global que se estabelece nos últimos anos. No Brasil, no final da década de 1980, estimou-se que o DM2 ocorria em cerca de $8 \%$ da população residente em áreas metropolitanas brasileiras. A intolerância à glicose era igualmente de $8 \%{ }^{34}$. Estimativas mais atuais revelam que cerca de $12,1 \%$ da população apresente diabetes ${ }^{35}$. 
Tabela 1. Perfil clínico-ocupacional por frequência nos trabalhadores de condomínio avaliados em ambulatório de Medicina Geral.

\begin{tabular}{|c|c|c|}
\hline & $\begin{array}{c}\text { Total } \\
(n=380)\end{array}$ & $\begin{array}{c}\text { Frequência } \\
(\%)\end{array}$ \\
\hline \multicolumn{3}{|l|}{ Ocupação } \\
\hline Portaria & 212 & 55,8 \\
\hline Serviços de limpeza* & 141 & 37,1 \\
\hline Zeladoria & 27 & 7,1 \\
\hline \multicolumn{3}{|l|}{ Sexo } \\
\hline Feminino & 182 & 47,9 \\
\hline Masculino & 198 & 52,1 \\
\hline \multicolumn{3}{|l|}{ Faixa etária (anos) } \\
\hline $20-29$ & 9 & 2,4 \\
\hline $30-39$ & 63 & 16,6 \\
\hline $40-49$ & 126 & 33,1 \\
\hline $50-59$ & 113 & 29,7 \\
\hline$\geq 60$ & 69 & 18,2 \\
\hline \multicolumn{3}{|l|}{ Hipertensão arterial sistêmica } \\
\hline Sim & 150 & 39,5 \\
\hline Não & 230 & 60,5 \\
\hline \multicolumn{3}{|l|}{ Diabetes tipo 2} \\
\hline Sim & 41 & 10,8 \\
\hline Não & 339 & 89,2 \\
\hline \multicolumn{3}{|l|}{ Pré-diabetes } \\
\hline Sim & 28 & 7,4 \\
\hline Não & 352 & 92,6 \\
\hline \multicolumn{3}{|l|}{ Tabagismo } \\
\hline Sim & 46 & 12,1 \\
\hline Não & 334 & 87,9 \\
\hline \multicolumn{3}{|l|}{ Colesterol total } \\
\hline$<240 \mathrm{mg} / \mathrm{dL}$ & 246 & 64,7 \\
\hline$\geq 240$ mg/dL ou uso de hipolipemiante & 134 & 35,3 \\
\hline \multicolumn{3}{|l|}{ Evento cardiovascular prévio } \\
\hline Sim & 6 & 1,6 \\
\hline Não & 374 & 98,4 \\
\hline \multicolumn{3}{|l|}{ Peso } \\
\hline Normal & 75 & 19,8 \\
\hline Sobrepeso & 162 & 42,6 \\
\hline Obesidade & 143 & 37,6 \\
\hline \multicolumn{3}{|l|}{ Sedentarismo } \\
\hline Sim & 304 & 80 \\
\hline Não & 76 & 20 \\
\hline
\end{tabular}

*Faxina e auxiliar de limpeza.
A faixa etária pesquisada nos profissionais foi predominantemente acima dos 40 anos (81\%). Trata-se de fator de risco não modificável e é constatado que o avançar da idade pode contribuir para maior adoecimento e ocorrência de complicações relacionadas às DCV.

O tabagismo esteve presente em $12,1 \%$ dos indivíduos. Esse dado segue a tendência de queda do número de fumantes no Brasil de acordo com os últimos inquéritos ${ }^{26,28}$. A meta do Ministério da Saúde é chegar a 9\% até 2022.

Destaca-se a elevada ocorrência de excesso de peso (sobrepeso e obesidade), ainda que seja analisada cada categoria profissional separadamente. No presente estudo, a prevalência foi de $80,2 \%$, com $37,6 \%$ do total avaliado situados na faixa de obesidade. Esses números estão bem acima quando comparados à população geral ${ }^{17,18}$.

Do mesmo modo, a taxa de sedentarismo também esteve muito elevada. Valores acima de $50 \%$ já foram reportados ${ }^{36,37}$, enquanto que na pesquisa atual foi de $80 \%$. Já foi demonstrado que a atividade física pode proteger contra as DCV bem como proporcionar redução de níveis pressóricos e no peso corporal, por exemplo ${ }^{38}$.

A hipercolesterolemia foi documentada em $35,3 \%$ dos indivíduos pesquisados. Estudos brasileiros que estabeleceram o mesmo nível de corte para diagnóstico relataram prevalência de 5,6 a 24,4\%, dependendo da população estudada ${ }^{39,40}$.

\section{DISCUSSÃo}

Para verificar se houve associação entre os fatores de risco de DCV e as ocupações estudadas, aplicou-se o teste $\chi^{2}$, seguido do cálculo da odds ratio (OR) e dos intervalos de confiança de $95 \%$ (IC95\%). Os resultados são apresentados na Tabela 2. A associação entre os fatores de risco de DCV e as ocupações estudadas é significativa se valor $\mathrm{p}<0,05$, o que ocorreu para os fatores de risco sedentarismo $(p=0,023)$, peso normal $(p=0,047)$ e HAS ( $p=0,046)$. Para o fator de risco sedentarismo, a categoria dos que executam "Serviços de Limpeza" (IC95\% 1,20-3,80) apresenta uma prevalência maior de sedentários em relação aos que ficam na "Portaria", e não há diferença em relação ao sedentarismo entre estes trabalhadores e os da "Zeladoria" (IC95\% 0,64-5,79).

Para o fator de risco peso normal, a categoria dos que executam "Serviços de Limpeza" (IC95\% 1,14-3,27) 
Tabela 2. Prevalência de fatores de risco de doenças cardiovasculares de acordo com as ocupações estudadas.

\begin{tabular}{|c|c|c|c|}
\hline \multirow[t]{2}{*}{ Total $(n=380)$} & Portaria & $\begin{array}{c}\text { Serviços de } \\
\text { limpeza* }\end{array}$ & Zeladoria \\
\hline & 212 & 141 & 27 \\
\hline \multicolumn{4}{|l|}{ Sedentarismo } \\
\hline Prevalência (\%) & 75,00 & 93,61 & 85,18 \\
\hline OR & 1,00 & 2,14 & 1,92 \\
\hline (IC95\%) & & $(1,20-3,80)$ & $(0,64-5,79)$ \\
\hline Valor p & 0,023 & & \\
\hline \multicolumn{4}{|l|}{ Peso normal } \\
\hline Prevalência (\%) & 15,56 & 26,24 & 18,51 \\
\hline OR & 1,00 & 1,93 & 1,23 \\
\hline (IC95\%) & & $(1,14-3,27)$ & $(0,44-3,49)$ \\
\hline Valor p & 0,047 & & \\
\hline \multicolumn{4}{|l|}{ Sobrepeso } \\
\hline Prevalência (\%) & 45,75 & 38,29 & 40,74 \\
\hline OR & 1,00 & 0,74 & 0,82 \\
\hline (IC95\%) & & $(0,48-1,14)$ & $(0,36-1,84)$ \\
\hline Valor p & 0,374 & & \\
\hline \multicolumn{4}{|l|}{ Obesidade } \\
\hline Prevalência (\%) & 38,67 & 35,46 & 40,74 \\
\hline OR & 1,00 & 0,87 & 1,09 \\
\hline (IC95\%) & & $(0,56-1,36)$ & $(0,48-2,46)$ \\
\hline Valor p & 0,781 & & \\
\hline \multicolumn{4}{|l|}{ Tabagismo } \\
\hline Prevalência (\%) & 12,73 & 8,49 & 3,70 \\
\hline OR & 1,00 & 0,87 & 1,09 \\
\hline (IC95\%) & & $(0,53-1,90)$ & $(0,03-2,02)$ \\
\hline Valor p & 0,38 & & \\
\hline \multicolumn{4}{|l|}{ HAS } \\
\hline Prevalência (\%) & 42,92 & 31,91 & 51,85 \\
\hline $\mathrm{OR}$ & 1,00 & 0,62 & 1,43 \\
\hline (IC95\%) & & $(0,40-0,97)$ & $(0,37-4,91)$ \\
\hline Valor p & 0,046 & & \\
\hline \multicolumn{4}{|l|}{ Diabetes tipo 2} \\
\hline Prevalência (\%) & 12,73 & 7,80 & 11,11 \\
\hline OR & 1,00 & 0,58 & 0,86 \\
\hline (IC95\%) & & $(0,28-1,21)$ & $(0,24-3,04)$ \\
\hline Valor p & 0,342 & & \\
\hline \multicolumn{4}{|c|}{ Hipercolesterolemia } \\
\hline Prevalência (\%) & 33,96 & 34,75 & 48,14 \\
\hline OR & 1,00 & 1,03 & 1,80 \\
\hline (IC95\%) & & $(0,66-1,62)$ & $(0,81-4,05)$ \\
\hline Valor p & 0,34 & & \\
\hline
\end{tabular}

OR: odds ratio; IC95\%: intervalos de confiança de 95\%; HAS: hipertensão arterial sistêmica; *Faxina e auxiliar de limpeza. apresenta uma prevalência maior de indivíduos com peso normal em relação aos que ficam na "Portaria", e não há diferença em relação ao peso normal entre estes trabalhadores $\mathrm{e}$ os da "Zeladoria" (IC95\% 0,44-3,49). Para o fator de risco HAS, a categoria dos que executam "Serviços de Limpeza" (IC95\% -0,40-0,97) tem uma prevalência mais baixa de HAS em relação à categoria dos que ficam na "Portaria", e não há diferença em relação à prevalência de HAS entre estes trabalhadores e os da "Zeladoria” (IC95\% 0,37-4,91).

Todas as três categorias profissionais avaliadas apresentaram índices preocupantes de fatores de risco para DCV. Muitos dos indivíduos pesquisados não sabiam de sua condição de saúde e a maioria nunca havia recebido orientações a respeito de cuidados de saúde no trabalho ou por meio de programas em sua comunidade. Trata-se de primeiro estudo que visou identificar e quantificar riscos de agravo à saúde nesse ambulatório de trabalhadores.

\section{CONCLUSÕES}

O estudo demonstrou elevada ocorrência de doenças crônicas que elevam significativamente o risco de morte em trabalhadores de condomínio. Cabe salientar que são ocupações preenchidas por indivíduos de menor escolaridade e de baixo nível socioeconômico, elementos que contribuem para maior impacto de potenciais prejuízos causados pelos fatores estudados.

Outras categorias profissionais também já apresentaram possível associação entre fatores de risco de doença cardiovascular e trabalho: trabalhadores de saúde, de turnos, da indústria, motoristas, policiais e pilotos da aviação ${ }^{41}$. Um novo perfil patológico se estabelece gradativamente ao longo das décadas, com maior prevalência na população trabalhadora de agravos à saúde marcados pelas doenças crônicas ${ }^{42}$.

A frequência elevada dos fatores de risco de DCV deve estimular a adoção de estratégias para melhorar as condições de saúde dos trabalhadores. O maior conhecimento do perfil clínico-ocupacional de um grupo fornece subsídios para viabilizar o correto direcionamento de recursos nos programas de saúde, bem como aumenta a qualidade de vida no trabalho. Um dos melhores instrumentos para se avaliar a eficácia das ações sobre esses fatores de risco é por meio de programas de saúde, comparando resultados com base em percentuais reais de prevalência ${ }^{38}$. 
1. Mansur AP, Favarato D. Mortalidade por doenças cardiovasculares no Brasil e na região metropolitana de São Paulo: atualização 2011. Arq Bras Cardiol. 2012;99(2):755-61.

2. Banco Mundial. Enfrentando o desafio das doenças não transmissíveis no Brasil. Relatório No. 32576-BR. Brasília: Banco Mundial/Unidade de Gestão do Setor de Desenvolvimento Humano Região da América Latina e do Caribe; 2005.

3. Grundy SM, Pasternak R, Greenland P, Smith Jr S, Fuster V. Assessment of cardiovascular risk by use of multiple-risk-factor assessment equations: a statement for healthcare professionals from the American Heart Association and the American College of Cardiology. Circulation 1999;100(13):1481-92.

4. Collins R, Peto R, MacMahon S, Hepert P, Fiebach NH, Eberlein $\mathrm{KA}$, et al. Blood pressure, stroke, and coronary heart disease. Part 2, Short-term reductions in blood pressure: overview of randomised drug trials in their epidemiological context. Lancet. 1990;335(8693):827-38.

5. Hebert PR, Gaziano JM, Chan KS, Hennekens CH. Cholesterol lowering with statin drugs, risk of stroke, and total mortality. An overview of randomized trials. JAMA 1997;278(4):313-21.

6. Hermanson B, Omenn GS, Kronmal RA, Gersh BJ. Beneficial six-year outcome of smoking cessation in older men and women with coronary artery disease. Results from the CASS registry. N Engl J Med. 1988;319(21):1365-9.

7. Sociedade Brasileira de Cardiologia; Sociedade Brasileira de Hipertensão; Sociedade Brasileira de Nefrologia. VI diretrizes brasileiras de hipertensão. Arq Bras Cardiol. 2010; 95(1 supl.1):1-51.

8. Cesarino CB, Cipullo JP, Martin JFV, Ciorlia LA, Godoy MRP, Cordeiro JA, et al. Prevalência e fatores sociodemográficos em hipertensos de São José do Rio Preto - SP. Arq Bras Cardiol. 2008;91(1):31-5.

9. Rosário TM, Scala LCN, França GVA, Pereira MRG, Jardim PCBV. Prevalência, controle e tratamento da hipertensão arterial sistêmica em Nobres - MT. Arq Bras Cardiol. 2009;93(6):672-8.

10. Pereira M, Lunet N, Azevedo A, Barros H. Differences in prevalence, awareness, treatment and control of hypertension between developing and developed countries. J Hypertens. 2009;27(5):963-75.

11. Fein F, Scheuer J. Heart disease in diabetes mellitus: theory and practice. In: Rifkin H, Port D (Eds). Diabetes Mellitus. New York: Elsever, 1990:812-23.

12. Wild S, Roglic G, Green A, Sicree R, King H. Global prevalence of diabetes: estimates for the year 2000 and projections for 2030. Diabetes Care. 2004;27(5):1047-53

13. Sever PS, Dahlöf B, Poulter NR, Wedel H, Beevers G, Caulfield M, et al. Prevention of coronary and stroke events with atorvastatin in hypertensive patients who have average or lower-than-average cholesterol concentrations, in the Anglo-Scandinavian Cardiac Outcomes Trial - Lipid Lowering Arm (ASCOT-LLA): a multicentre randomised controlled trial. Drugs. 2004;64(Suppl 2):43-60.

14. LaRosa JC, Hunninghake D, Bush D, Criqui MH, Getz GS, Gotto AM $\mathrm{Jr}$, et al. The cholesterol facts. A summary of the evidence relating dietary fats, serum cholesterol, and coronary heart disease. A joint statement by the American Heart Association and the National Heart, Lung, and Blood Institute. The Task Force on Cholesterol Issues, American Heart Association. Circulation. 1990;81(5):1721-33.
15. Kannel WB, Castelli WP, Gordon T, McNamara PM. Serum cholesterol, lipoproteins, and the risk of coronary heart disease. The Framingham Study. Ann Intern Med. 1971;74(1):1-12.

16. Calle EE, Thun MJ, Petrelli JM, Rodriguez C, Heath CW Jr. Bodymass index and mortality in a prospective cohort of U.S. adults. N Eng J Med. 1999;341(15):1097-105.

17. Brasil. Ministério da Saúde. VIGITEL Brasil 2009: vigilância de fatores de risco e proteção para doenças crônicas por inquérito telefônico. Brasília: Ministério da Saúde; 2010.

18. Brasil. Ministério da Saúde, Ministério do Planejamento, Orçamento e Gestão, Instituto Brasileiro de Geografia e Estatística (IBGE). Pesquisa de orçamentos familiares 2008 - 2009: antropometria e estado nutricional de crianças, adolescentes e adultos no Brasil. Rio de Janeiro: IBGE; 2010.

19. World Health Organization. Global health risks: mortality and burden of disease attributable to selected major risks. Geneva: WHO; 2009.

20. World Health Organization. The global burden of disease: 2004 update. Geneva: WHO; 2008.

21. World Health Organization. A guide for population-based approaches to increasing levels of physical activity: implementation of the WHO Global Strategy on Diet, Physical Activity and Health. Geneva: WHO; 2007.

22. World Health Organization. WHO global report: mortality attributable to tobacco. Geneva: WHO; 2012.

23. World Health Organization. WHO Report on the Global Tobacco Epidemic, 2008: The MPOWER package. Geneva: WHO; 2008.

24. Brasil. Ministério da Saúde, Instituto Nacional do Câncer (Inca). Prevalência de tabagismo no Brasil: dados dos inquéritos epidemiológicos em capitais brasileiras. Rio de Janeiro: Inca; 2004.

25. Mackay J, Eriksen M. The tobacco atlas. Geneva: WHO; 2002.

26. Brasil. Ministério da Saúde, Instituto Nacional do Câncer (Inca). Programa Saber Saúde: buscando formar cidadãos conscientes. Atualidades em tabagismo e prevenção do câncer. Rio de Janeiro: Inca; 2001;10.

27. Brasil. Ministério do Planejamento, Orçamento e Gestão, Instituto Brasileiro de Geografia e Estatística (IBGE). Pesquisa nacional por amostra de domicílios - Tabagismo 2008. Rio de Janeiro: IBGE; 2009.

28. Brasil. Ministério da Saúde. VIGITEL Brasil 2011: vigilância de fatores de risco e proteção para doenças crônicas por inquérito telefônico. Brasília: Ministério da Saúde; 2012.

29. Angeluci FL, Copacheski FM, Cieslack JL. A qualidade de vida no ambiente de trabalho e a aplicação dos princípios relativos ao programa de controle médico de saúde ocupacional [Monografia]. Guarapuava: UEPG; 2005.

30. Alves EF. Programas e ações em qualidade de vida no trabalho. Interfacehs. 2011;6(1):60-78.

31. World Health Organization. Global recommendations on physical activity for health. Geneva: WHO; 2010.

32. R Development Core Team. R: A language and environment for statistical computing. Vienna: R Foundation for Statistical Computing. Disponível em: <http://www.r-project.org>.

33. Yunis $\mathrm{C}$, Krob HA. Status of health and prevalence on hypertension in Brazil. Ethn Dis. 1998;8(3):406-12.

34. Brasil. Ministério da Saúde. Cadernos de atenção básica n.o 16 Diabetes mellitus. Brasília: Ministério da Saúde; 2006. 
35. Torquato MT, Montenegro-Júnior RN, Viana LA, Souza RA, Lanna CM, Lucas JC et al. Prevalence of diabetes mellitus and impaired glucose tolerance in the urban population aged 30-69 years in Ribeirão Preto (São Paulo), Brazil. Sao Paulo Med J. 2003;121(6):224-30.

36. Pitanga FJG, Lessa I. Prevalência e fatores associados ao sedentarismo no lazer em adultos. Cad Saúde Pública. 2005;21(3):870-7.

37. Olbrich SRLR, Nitsche MJT, Mori NLR, Neto JO. Sedentarismo: prevalência e associação de fatores de risco cardiovascular. Rev Ciênc Ext. 2009;5(2):30-41.

38. Leon AS, Connett J, Jacobs-Junior DR, Rauramaa R. Leisuretime physical activity levels and risk of coronary heart disease and death. The Multiple Risk Factor Intervention Trial. JAMA 1987;258(17):2388-95.
39. Gus I, Fischmann A, Medina C. Prevalência dos fatores de risco da doença arterial coronariana no Estado do Rio Grande do Sul. Arq Bras Cardiol. 2002;78(5):478-83.

40. Siqueira AFA, Harima HA, Osiro K, Hirai AT, Gimeno SGA, Ferreira SRG, Japanese-Brazilian Diabetes Study Group. Distúrbios no perfil lipídico são altamente prevalentes em população nipo-brasileira. Arq Bras Endocrinol Metab. 2008;52(1),40-6.

41. Beltrão FLL, Pena PGL. Associação entre síndrome metabólica e saúde no trabalho. Rev Bras Med Trab. 2013;11(1):3-18.

42. Lacaz FAC. Qualidade de vida no trabalho e saúde/doença. Ciênc Saúde Coletiva. 2000;5(1):151-61.

Endereço para correspondência: Maurício Susumu Osawa - Rodovia Celso Garcia Cid, km 380 - Jardim Portal de Versalhes 1 - CEP: 86055-900 -

Londrina (PR), Brasil - E-mail: mauricioosawa@yahoo.com.br 